\title{
Lentiviral-mediated Smad4 RNAi induced anti-proliferation by p16 up-regulation and apoptosis by caspase 3 down-regulation in hepatoma SMMC-7721 cells
}

\author{
SHU HUANG ${ }^{1 *}$, FAMING ZHANG $^{1,2^{*}}$, LIN MIAO $^{1}$, HUIJIE ZHANG ${ }^{1}$, \\ ZHINING FAN ${ }^{1}$, XUEHAO WANG ${ }^{3}$ and GUOZHONG JI ${ }^{1}$
}

\begin{abstract}
${ }^{1}$ Institute of Digestive Endoscopy and Medical Center for Digestive Diseases, Second Affiliated Hospital of Nanjing Medical University, Nanjing 210011, ${ }^{2}$ State Key Laboratory of Cancer Biology and Institute of Digestive Diseases, Xijing Hospital, Fourth Military Medical University, Xi'an 710032, ${ }^{3}$ Department of Hepatobiliary Surgery, First Affiliated Hospital of Nanjing Medical University, Nanjing 210029, P.R. China
\end{abstract}

Received April 18, 2008; Accepted June 3, 2008

DOI: 10.3892/or_00000109

\begin{abstract}
Transforming growth factor- $\beta$ (TGF- $\beta$ )-Smad signaling pathway participates in the regulation of a variety of cellular activities. Unlike the high incidences of Smad4 mutation or deletion in pancreatic cancer and gastrointestinal cancers, Smad4 gene is seldom mutated or deleted in hepatocellular carcinoma (HCC). The role of TGF-ß-Smad4 signaling pathway in leading to carcinogenesis of liver cells remains unknown. In this study, we succeeded in silencing Smad4 using lentiviral-mediated Smad4 RNA interference (RNAi). We investigated the role of Smad4 in TGF- $\$ 1$-induced cell proliferation and apoptosis of HCC cell line SMMC-7721. We determined cell proliferation, apoptosis, and expression of p21, p16, p53 and caspase 3. Results showed that TGF-ß1 not only had a significant anti-proliferation effect but also induced cellular apoptosis in SMMC-7721 cells. These effects induced by TGF- $\beta 1$ were almost completely blocked by the knockdown of Smad4. Western blot analysis revealed that p16 was up-regulated and caspase 3 was activated by silencing of Smad4, and the expression of p21 and wild-type p53 were not affected. These results suggest that TGF-B1induced cell growth inhibition by up-regulating p 16 expression and cellular apoptosis by activating caspase 3 was Smad4-dependent. Additionally, the knock down of a
\end{abstract}

Correspondence to: Dr Guozhong Ji, Institute of Digestive Endoscopy and Medical Center for Digestive Diseases, Second Affiliated Hospital of Nanjing Medical University, Nanjing 210011, P.R. China

E-mail: jgzzl@163.com

${ }^{*}$ Contributed equally

Key words: transforming growth factor- $\beta$, smad4, hepatocellular carcinoma, RNA interference, proliferation, apoptosis, p16, caspase 3 specific gene using lentiviral-mediated RNAi appears to be a promising tool and strategy for analyzing endogenous gene function.

\section{Introduction}

Transforming growth factor (TGF) Bs are polypeptides that regulate several cellular functions, including cell growth and differentiation, motility, extra cellular matrix production, and immune functions (1-4). TGF-ßs have three mammalian isoforms, TGF- $\$ 1,-\beta 2$ and $-\beta 3$ each with distinct functions in vivo. All three TGF-ßs use the same receptor signaling system. TGF- $\beta$ has three receptors, type I (RI), type II (RII) and type III (RIII), RIII (also called $\beta$ glycan) binds two TGF- $\beta$ polypeptides and recruits TGF- $\beta$ to RII and intensifies the TGF- $\beta$ signaling pathway (1-5). TGF- $\beta$ binding to RII stimulates recruitment and transphosphorylation of RI. RI, in turn, phosphorylates Smad2 and 3, which form an oligomeric complex with the Smad4 protein. Smad4 has a central role in the process of TGF- $ß 1$ signaling pathway (1-3,5-7). Deregulation of TGF- $\beta$ expression or signaling has been implicated in the pathogenesis of a variety of diseases, including cancer and fibrosis.

There is growing evidence that in the later stages of cancer development TGF- $\beta$ is actively secreted by tumor cells and does not merely act as a bystander but rather contributes to cell growth, invasion, and metastasis and decreases host-tumor immune responses cell. Studies have suggested that TGF- $\beta 1$ may be a useful serologic and urinary marker in detecting HCC (8-10). Moreover, TGF- 11 was overexpressed in HCC (11-13), the expression level of TGF-ß1 was correlated with the histological differentiation in HCC tissue (13). The evidence proves that TGF- $\beta 1$ plays an important role in HCC. Elucidation of alteration of TGF- $\beta 1$ signaling will help in further understanding the molecular mechanisms of hepatocarcinogenesis. Many investigations have revealed that the major factors of TGF-ß1-Smad4 signaling pathway, such as receptors and Smad proteins, function in gastro- 
intestinal, pancreas and breast cancer (14-20). However, there is not enough evidence to establish the mechanisms or consequence of aberrant regulation of Smad4 in HCC, some of which are even conflicting (14-16,20,21). Ijichi et al examined 8 hepatic cancer cell lines and did not observed impaired signaling of TGF-ß1-Smad4 pathway, suggesting that this pathway did not function as a tumor suppressor in hepatic carcinogenesis (16). Yakicier et al studied 35 HCC and non-tumor liver tissues and found 2 tumor tissues displayed somatic mutations in Smad4 gene, demon-strating that TGF-ß1-Smad4 pathway was altered in hepatocellular carcinoma (20). Using protein microarrays, Tannapfel et al discovered that the expression of Smad4 was down-regulated (21). However, Torbenson et al reported that Smad4 was overexpressed in hepatocellular carcinoma (14). Therefore, the roles of TGF-31-Smad4 signaling pathway in HCC need further study.

In the present study, we investigated the function of TGF-ß1-Smad4 signaling pathway in SMMC-7721 cells. We applied lentiviral-mediated Smad4 RNAi to knockdown Smad4 expression. We observed the effects of TGF- $\beta 1$ on the cell growth and apoptosis. Then, we examined the role of p16, 21,53 and caspase 3 in the effects of TGF- 31 . Our results demonstrated that TGF-ß1-induced cell growth inhibition by up-regulating the expression of p16 and cellular apoptosis by activating caspase 3 in a Smad4-dependent manner.

\section{Materials and methods}

Cell culture. Human hepatocellular carcinoma cell line SMMC-7721 (purchased from the Institute of Biochemistry and Cell Research, China Life Science Academy, Shanghai, China) was cultured in DMEM supplemented with $10 \%$ fetal bovine serum (containing $100 \mathrm{U} / \mathrm{ml}$ of penicillin and streptomycin, respectively) at $37^{\circ} \mathrm{C}$ in a humidified atmosphere of $5 \%$ of $\mathrm{CO}_{2}$. Exponentially growing cells were used for the experiments. The cells were treated with TGF- $\beta 1$ (Sigma, MO) at the concentration of $100 \mathrm{ng} / \mathrm{ml}$.

Design and cloning of lentiviral shRNA vectors. The target siRNAs against human Smad4 (GenBank, gi: 34147555) for RNAi were designed as: siRNA1: 5'-GCACAAGGTTGGTT GCTAA-3', siRNA2: 5'-CCAGCTACTTACCATCATA-3', siRNA3: 5'-GTACTTCATACCATGCCGA-3', Control sequence of RNAi: 5'-TTCTCCGAACGTGTCACGT-3'. The siRNA3 was the published sequence designed by Jazag et al (6). In our previous study, we confirmed that siRNA3 was the optimal target siRNA in the above 3 sequences (22). Lentiviral vector system was purchased from Tronolab (http://tronolab.com/lentivectors.php). This vector system including 3 plasmids: pLVTHM vector, pCMV-dR8.74 and pMD2G. The targeting sequence was subcloned into the pLVTHM vector, which contained $\mathrm{H} 1$ promoter and green fluorescent protein (GFP). The resulting lentiviral vector containing Smad4 shRNA was named RNAi-Smad4, and the sequence was confirmed by PCR and sequencing. Lentiviral vector containing negative control sequence of Smad4 shRNA (RNAi-NC) containing the non-silencing sequence was used as infection control.
Lentiviral vector production and cell infection. Lentiviral vectors were produced by transient transfection of HEK293 cells according to standard protocol. HEK293 cell line was supplemented with $10 \%$ fetal calf serum and, when subconfluent, transfected with $1800 \mu 1$ DNA solution: $20 \mu \mathrm{g} \mathrm{LV}-$ shSmad4, $15 \mu \mathrm{g}$ pCMV-dR8.74 and $7.5 \mu \mathrm{g}$ pMD2G. All virus stocks were produced by calcium phosphate-mediated transfection. After $48 \mathrm{~h}$ post-transfection cell supernatants, containing viral particles, were filtered using $0.45 \mu \mathrm{m}$ Steriflip vacuum filtration system (Millipore) and concentrated by ultracentrifugation at $25,000 \mathrm{rpm}$ at $4^{\circ} \mathrm{C}$. The titer of virus was tested according to the expression level of GFP. The day before infection the SMMC-7721 cells were seeded on dishes with the conference of $30-40 \%$. On the day of infection the SMMC-721 cells were infected by packaged lentiviral production. The parallel SMMC-721 cells without infection were observed at the same time. Cells were cultured in normal growth medium for $72 \mathrm{~h}$ after infection. RNAi-NC as infection control was performed as above.

Cell growth analysis. The cellular growth was evaluated by MTT colorimetric assay. Briefly, the cells were thoroughly washed with PBS and then seeded in a 96-well plate $\left(2 \times 10^{4}\right.$ cells/well). After $24 \mathrm{~h}, 20 \mu \mathrm{l}$ of MTT solution $(1 \mathrm{mg} / \mathrm{ml})$ was added to each well and mixed with the cells. Four hours later, $150 \mu 1$ of DMSO was added into each well and incubated with the cells for $10 \mathrm{~min}$. Then optical density at $490 \mathrm{~nm}$ was determined using an ELISA reader (Bio-TEK Instruments, USA). The TGF- $B 1$ stimulation of dose- and time-dependent effects was determined.

Cell apoptosis analysis. Cell apoptosis was assayed with terminal deoxynucleotidyltransferase-mediated dUTP-biotin nick end labeling (TUNEL) assay and propidium iodide (PI) staining. TUNEL assay was performed using a kit (Roche, USA) according to manufacturer's instructions. Briefly, DNA ends were tagged with fluorescein-labled dUTP using terminal deoxynucleotidyl transferase by incubating the samples at $37{ }^{\circ} \mathrm{C}$ in a humidified chamber. Cells after treatment with TGF- $\beta 1$ for $36 \mathrm{~h}$ were washed with PBS, fixed with $2 \%$ paraformaldehyde. The slides with cells were then washed 3 times in distilled water, immersed in penetration liquid and incubated on ice for $2 \mathrm{~min}$. Then the slides were washed 3 times in $\mathrm{PBS}$, and $3 \% \mathrm{H}_{2} \mathrm{O}_{2}$ was added into the chamber for $30 \mathrm{~min}$ at room temperature. The slides were rinsed twice in PBS, immersed in TdT reaction liquid, incubated for $30 \mathrm{~min}$ at $37^{\circ} \mathrm{C}$, washed in PBS for $5 \mathrm{~min}$, and rinsed in distilled water for $30 \mathrm{sec}$. Then slides were dealt with prolong anti-fade reagent kit. Morphologic changes were observed under fluorescent microscopy. Sections were also stained with PI to label DNA in the sample. Blue excitation was used to assess the orange-red PI fluorescence. Fluorescence images were taken using Kodak Gold 1000 ASA color film with automatic exposure. Positively stained cells obtained by the respective apoptosis assay were quantified. At least 300 cells were counted for each slide in 5 random fields.

Western blot analysis. Samples of cell extracts were homogenized in SDS-PAGE sample buffer containing 2\% SDS. After being heated at $80^{\circ} \mathrm{C}$ for $5 \mathrm{~min}$ and clarified by 
A

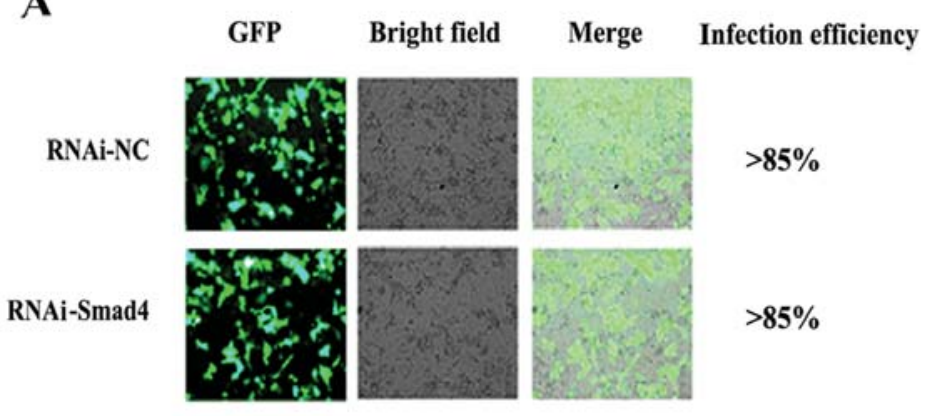

B

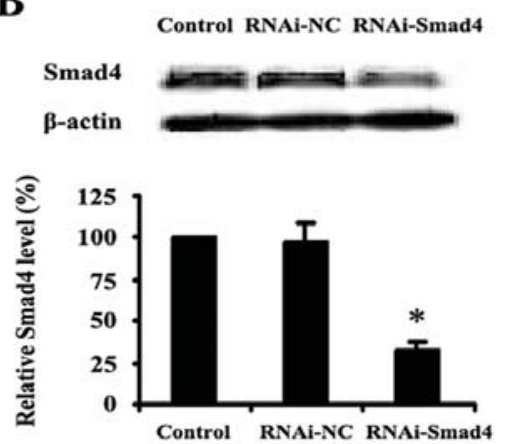

Figure 1. Knock down of Smad4 in SMMC-7721 cells by lentiviral-mediated RNAi. The validation of the effective siRNA against human Smad4 in the present study was determined in our previous study. SMMC-7721 cells were infected with lentiviral RNAi vector of the negative control (RNAi-NC) and lentiviral RNAi vector of Smad4 shRNA (RNAi-Smad4) for $36 \mathrm{~h}$, respectively. (A) Infection efficiency was assayed according to the expression of GFP. (B) Expression of Smad4 protein was measured by Western blot analysis. All values were expressed in relation to that of the control cells (cells without infection). The summary data are from three independent experiments. ${ }^{*} \mathrm{P}<0.05$ vs. RNAi-NC.

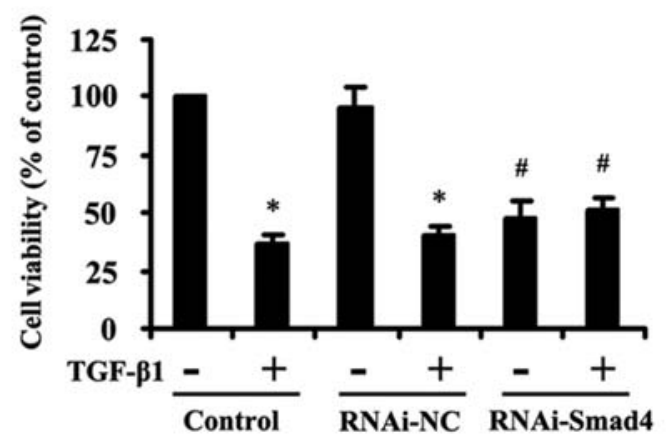

Figure 2. Effect of lentiviral-mediated Smad4 RNAi on TGF-B1-induced anti-proliferation. SMMC-7721 cells were infected by RNAi-NC and RNAiSmad4 for $24 \mathrm{~h}$, respectively. Cells in each group (control cells, RNAi-NC and RNAi-Smad4) were treated or untreated by $100 \mathrm{ng} / \mathrm{ml}$ TGF- $\beta 1 \mathrm{for} 36 \mathrm{~h}$, which were determined by dose- and time-dependent effects (figures not shown). The cell viability was measured by MTT assay at $490 \mathrm{~nm}$ optical density. The summary data were from three independent experiments. ${ }^{*} \mathrm{P}<0.05$ vs. the related TGF- 11 (-) stimulated cells. ${ }^{\#} \mathrm{P}<0.05$ vs. RNAi-NC infected cells with TGF-ß1 (+) or TGF-ß1 (-) stimulation.

spinning in a microcentrifuge at $12,000 \mathrm{rpm}$ and room temperature for $5 \mathrm{~min}$, the total protein extracts were resolved by polyacrylamide gel using the Laemmli buffer system. The resulting gels were stained with Coomassie Blue R-250 to reveal the resolved protein bands. Gels were electrically transferred to nitrocellulose membranes. After blocking in TBS containing 5\% non-fat-milk, the membranes were incubated with mouse monoclonal antibody against caspase 3 (Santa Cruz) (1:1000 in TBS containing 5\% non-fat milk), and rabbit polyclonal antibodies against Smad4 (Upstate) (1:1000), p16 (Cell Signaling) (1:1000), p21 (Cell Signaling) (1:1000), wild-type p53 (Santa Cruz) (1:1000), and B-actin (Bost, P.R. China) (1:2000). The membranes were then washed with TBS containing $0.05 \%$ Tween-20, and incubated with peroxidase-conjugated anti-mouse or anti-rabbit IgG secondary antibody (Sigma) (1: 2000) and washed again. The blots were developed in ECL substrate solution (Sigma) to reveal the immunoreactive bands. The protein expression was analyzed by Bandscan software and normalized by the quantity of $\beta$-actin in the same membrane.

Statistical analysis. Statistical analysis was performed using SPSS 11.0. Data were expressed as mean \pm SD. One-way analysis of variance (ANOVA) followed by LSD-test was used to assess significant differences among groups. Two independent samples test was used to assess the differences between 2 groups. Differences were considered significant at $\mathrm{P}<0.05$.

\section{Results}

Inhibition of Smad4 expression by RNA interference. In an initial study (22) we designed the siRNAs against human Smad4 and described the validation of the effective targeting site, these were used in the present study. We now performed Smad4 knockdown of SMMC-7721 cells by lentiviralmediated RNAi. As shown in Fig.1A, infection efficiency of lentiviral RNAi vectors of Smad4 (RNAi-Smad4) and the negative control (RNAi-NC) in SMMC-7721 cells was $>85 \%$. No significant inhibition on protein expression of Smad4 was observed in cells infected with RNAi-NC. However, Smad4 was knocked down significantly by the infection with RNAiSmad4 (Fig. 1B). These results indicated that lentiviralmediated RNAi could effectively inhibit Smad4 expression.

Lentiviral-mediated Smad4 RNAi induced anti-proliferation of SMMC-7721 cells. We examined the cell growth of SMMC-7721 cells infected by RNAi-Smad4 and non-specific control oligonucleotides (RNAi-NC). The TGF-ß1 stimulation of time-dependent effect determined that the inhibition effect was enhanced with prolonged exposure time with TGF- 31 stimulation (detailed data and figures not shown). We further measured and displayed the cell growth of SMMC-7721 cells with 72 h- TGF-ß1 stimulation. As shown in Fig. 2, TGF-ß1 had a significant anti-proliferation effect on parent SMMC7721 cells (without infection) or cells infected with RNAi-NC, respectively, however, there was no significant differences between RNAi-Smad4 infected cells with and without TGF- 31 stimulation. Compared with cells with RNAi-NC infection, 
the cell growth was repressed by RNAi-Smad4 when SMMC-7721 cells were stimulated with or without TGF- 11 stimulation, respectively (Fig. 2).

Role of lentiviral-mediated Smad4 RNAi on the expression of p16 and $p 21$. To investigate the mechanism that Smad4 represses TGF-ß1-induced growth inhibition of SMMC-7721 cells, we studied the expression of p16 and 21 (Fig. 3). The protein p16 was highly expressed in TGF- 11 -treated cells, including control cells and RNAi-NC infection cells. As for the cells infected with RNAi-Smad4, p16 expression was not affected significantly, indicating that the up-regulation effect of TGF-B1 on p16 expression was blocked by Smad4 silencing. As shown in Fig. 4B, unlike p16, the expression of p21 in each group was not regulated by Smad4 silencing when cells were stimulated by TGF- $\beta 1$ or not.

Lentiviral-mediated Smad4 RNAi-induced apoptosis. We studied the effect of lentiviral-mediated Smad4 RNAi on apoptosis of SMMC-7721 cells. Apoptosis analysis was measured by PI staining and TUNEL assay. As shown in Fig. 4, infection with LV-RNAi-Smad4 caused significant apoptosis when cells were treated by TGF- $\beta 1$, which suggested that TGF-ß1-induced cell apoptosis in SMMC-7721 was dependent on Smad4.

Role of lentiviral-mediated Smad4 RNAi on the expression of p53 and caspase 3. As shown in Fig. 5A, wild-type p53 was not regulated by silencing of Smad4 or TGF- $\beta 1$ stimulation in SMMC-7721 cells. These results implied that p53 should not be associated with TGF-B1-induced apoptosis in SMMC7721 cells. However, pre-caspase 3 was cleaved to the smaller fragment spliced-caspase 3 when Smad4 in cells were not knocked down (Fig. 5B), suggesting that caspase 3 was activated by TGF- 11 , Smad4 RNAi could significantly inhibit activation of caspase 3. Caspase 3 should be regulated by TGF- $\beta 1-$ Smad4 signaling pathway, and be involved in TGF-ß1-induced apoptosis in SMMC-7721 cells.

\section{Discussion}

At present, the role of TGF-B1-Smad4 signaling pathway in HCC is unknown. This study aimed to study the effect of Smad4 on apoptosis and proliferation of SMMC-7721 cell line and explore the possible mechanism. RNAi against Smad4 gene was designed to knock down its expression in SMMC7721 cells. The Smad4 shRNA lentiviral vector was constructed successfully. The lentiviral-mediated RNAi against Smad4 inhibited Smad4 expression effectively. We found that TGF- $\beta 1$ had a significant anti-proliferation effect on SMMC-7721 cells, which could be almost reversed by the Smad4 RNAi, suggesting that TGF- 11 -induced growth inhibition of SMMC-7721 cells was Smad4-dependent. Our results were consistent with a previous study on the SMMC7721 cell line (23). It has been well documented that cell proliferation depends on the cellular division cycles. Therefore, to investigate the cell cycle associated proteins regulated by Smad4 it is useful to explain the mechanism. TGF-ßs potently inhibits cell cycle progression at the G1 phase $(1,24)$, the G1 checkpoint can be viewed as a master
A
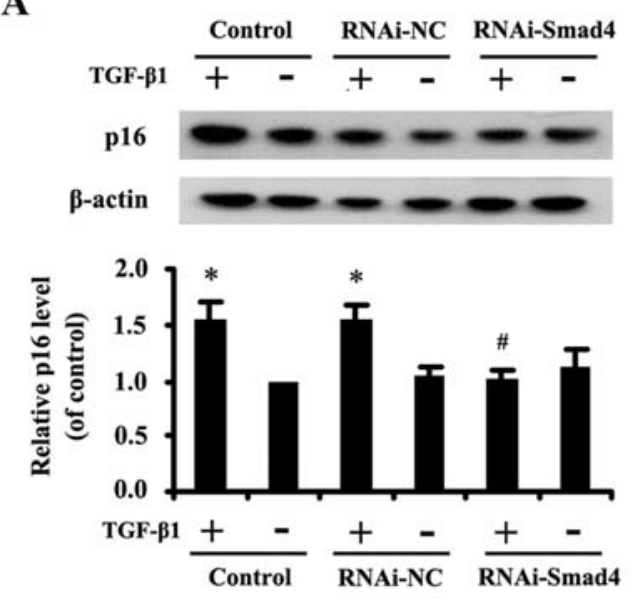

B
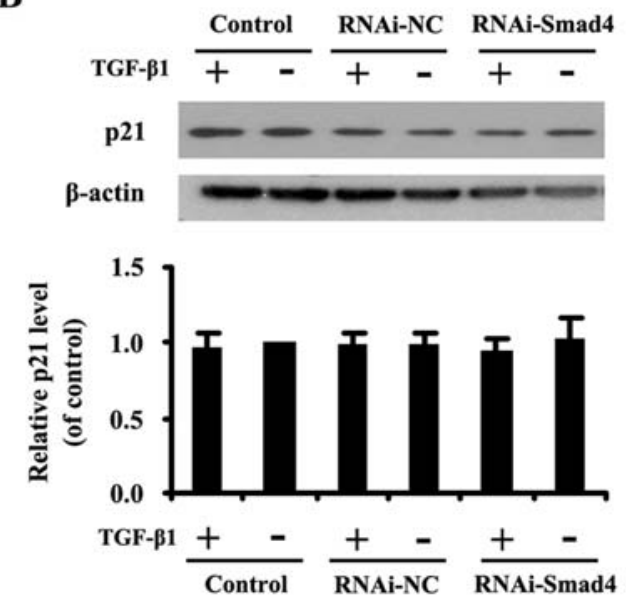

Figure 3 . Regulation of p16 and p21 by TGF-ß1-Smad4 signaling pathway in SMMC-7721 cells. SMMC-7721 cells were infected by RNAi-NC or RNAi-Smad 4 for $24 \mathrm{~h}$. Cells were treated or untreated by $100 \mathrm{ng} / \mathrm{ml}$ TGF- 31 for $36 \mathrm{~h}$, respectively. Expression of p16 (A) and p21 (B) was measured by Western blot analysis. The relative level of p16 or p21 was related to the corresponding control cells without TGF- $\$ 1$ stimulation. The summary data were from three independent experiments. ${ }^{*} \mathrm{P}<0.05 \mathrm{vs}$. the related group untreated by TGF-ß1. "P<0.05 vs. RNAi-NC infected cells with TGF-ß1 (+) or TGF-ß1 (-) stimulation.

checkpoint of the mammalian cell cycle $(1,24)$. Regulation of the $G_{1}$ phase of the cell cycle involves many different families of cyclins, cyclin-dependent kinases (CDKs) and cyclindependent kinase inhibitors (CKIs) (25). p16 belongs to the INK4 family of CKIs and specifically inhibits CDK4/6 by preventing binding of the activating cyclin subunits to arrest cells in $\mathrm{G}_{0} / \mathrm{G}_{1}$ phase (1). p21, a CKI of CIP/KIP family, unlike p16, binds to a number of cyclin/CDK complexes, inhibits kinase activities and induces cell cycle arrest at $\mathrm{G}_{0}-\mathrm{G}_{1}$ phase $(20,21,26)$. As revealed by previous experiments, the expression of p16 and 21 were abnormal in $\operatorname{HCC}(27,28)$. Grau et al reported that p21 was downstream of Smad4 (29), but a recent study reported that it was up-regulated in a Smad4-independent manner (30). Hui et al reported that p16 protein was absent from 3 of 6 cell lines (50\%) and 11 of 32 primary tumors $(34 \%)$, but present in non-cancerous tissues, indicating that p16 is involved in hepatocarcinogenesis (20). In this study, p16 protein was up-regulated significantly by 

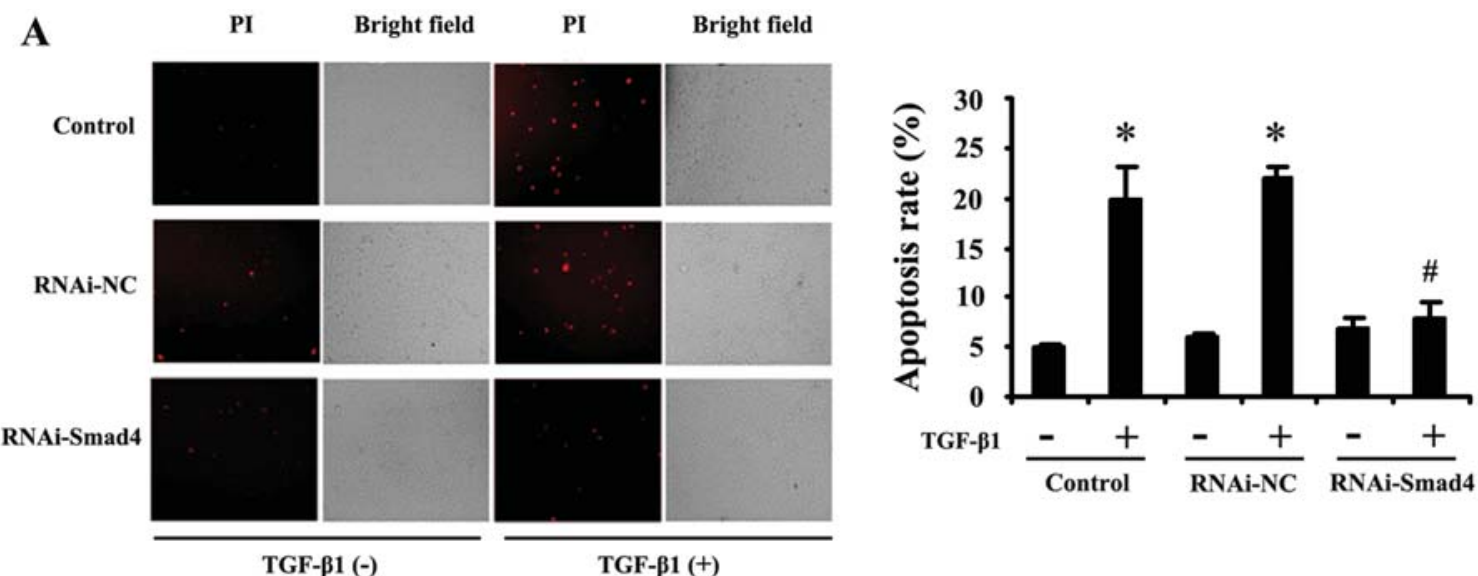

B

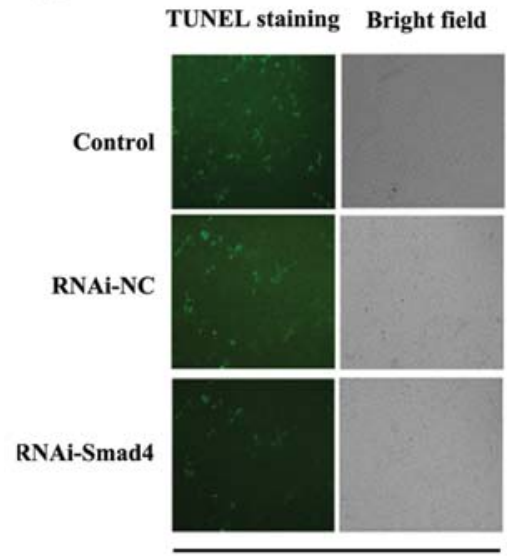

TGF-p1 (-)

\section{TUNEL staining Bright field}

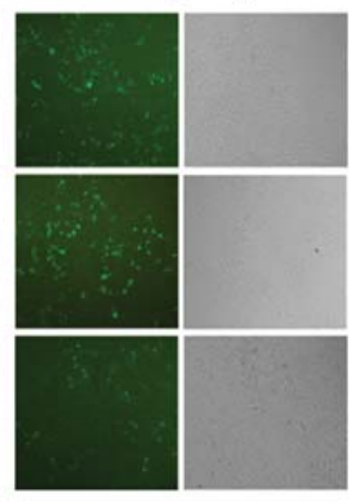

TGF-B1 (+)

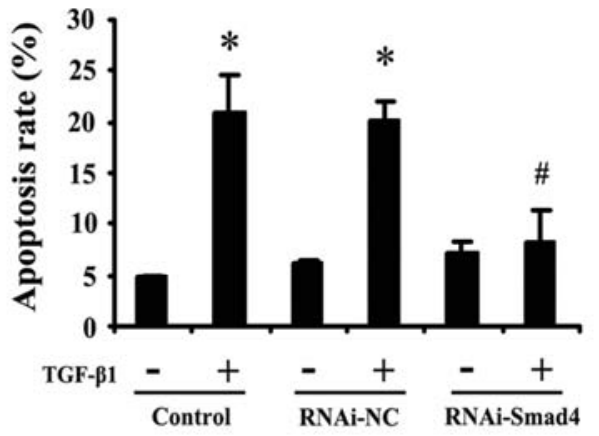

Figure 4. Effect of lentiviral-mediated Smad4 RNAi on TGF-B1-induced cell apoptosis. SMMC-7721 cells were infected by RNAi-NC or RNAi-Smad4 for $24 \mathrm{~h}$, and then incubated in the absence or presence of $100 \mathrm{ng} / \mathrm{ml}$ TGF- $\beta 1$ for $36 \mathrm{~h}$. Cellular apoptosis was assayed by PI staining (A) and TUNEL staining (B). The apoptosis rates of cells shown in (A, right) and (B, right) suggested similar results. ${ }^{*} \mathrm{P}<0.05$ vs. the related cells with TGF- 31 (-) stimulation. ${ }^{\#} \mathrm{P}<0.05$ vs. RNAi-NC infected cells with TGF-ß1 (+) stimulation. The results represent the average of three individual experiments.

A

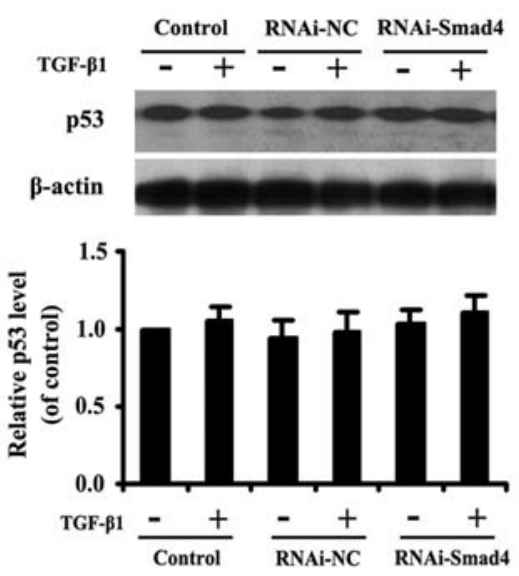

B

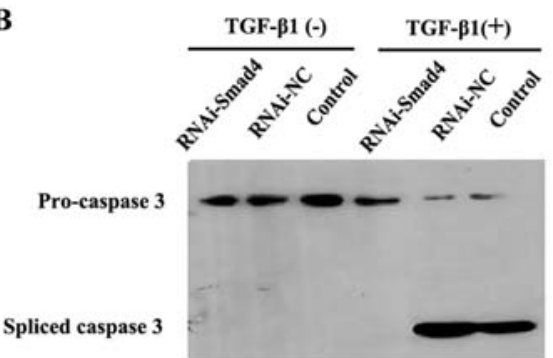

TGF- 1 , and this effect was blocked by RNAi against Smad4. However, neither the treatment of TGF- 31 nor the silence of Smad4 affected the expression of p21 in SMMC7721 cells. These results suggest that $\mathrm{p} 16$, instead of $\mathrm{p} 21$, acts as a downstream gene of Smad4, participating in the signal transduction of TGF- 31 in SMMC-7721 cells. p16 may be involved in TGF- $\beta 1$-induced cell degrowth in a Smad4-dependent manner.

TGF- $\beta 1$ has been shown to induce apoptosis in primary hepatocyte cultures and hepatoma cells in vitro as well as in the regressing liver (31). However, the mechanism of TGFß1-induced apoptosis in hepatoma cells needs further study.

Figure 5. Regulation of p53 and caspase 3 by TGF-ß1-Smad4 signaling pathway in SMMC-7721 cells. SMMC-7721 cells were infected by RNAi$\mathrm{NC}$ or RNAi-Smad4 for $24 \mathrm{~h}$. Cells then were treated or untreated by 100 $\mathrm{ng} / \mathrm{ml}$ TGF- $\beta 1$ for $36 \mathrm{~h}$, respectively. Expression of p53, pre-caspase 3 and spliced caspase 3 was measured by Western blot analysis. (A) The expression of p53 was not regulated by Smad4 silencing or TGF- 31 stimulation. The results represent the average of three individual experiments. (B) Spliced caspase 3 was highly expressed in RNAi-NC infected cells and control cells with TGF-ß1 (+) stimulation, however, there was no spliced caspase 3 expression in RNAi-Smad4 infected cells with TGF-ß1 (+) stimulation or in any cells with TGF-ß1 (-) stimulation. 
In the present study, we found that TGF- 31 induced SMMC7721 cell apoptosis and this effect was reversed by Smad4 silencing, indicating that TGF- $\beta 1$-induced apoptosis was dependet on Smad4 in SMMC-7721 cells. Since it is known that wild-type p53 protein can induce cell apoptosis, and mutation of p53 was observed with a high incidence in HCC $(27,32)$, the present study explored whether the wild-type p53 plays an important role in TGF- $\beta 1$-induced apoptosis. The results showed that TGF-ß1-induced apoptosis of SMMC7721 cells was not companied with the change of wild-type p53 expression, demonstrating that p53 may not be regulated by the TGF- $31-$ Smad4 signaling pathway.

Caspases, a group of homologous proteins, belong to cysteine proteases family. Caspase 3 is a downstream effector cysteine protease in the apoptotic pathway, and it is regarded as one of the main executors of apoptosis (33). The activation of caspase 3 plays important roles in the hepatocellular carcinomas apoptosis $(33,34)$. In this study, we observed that caspase 3 was activated in SMMC-7721 cells by TGF- $\beta 1$ and produced spliced caspase 3 . This activation was inhibited by lentiviral-mediated Smad4 silencing. These results indicated that caspase 3 regulated by Smad4 may mediate the apoptosis of SMMC-7721 cells induced by TGF-ß1. It is possible that, as the result of Smad4 knockdown, this triggers the release of cytochrome $\mathrm{C}$, leading to caspase- 3 activation and finally apoptosis.

Taken together, the present study revealed that in hepatocellular carcinoma cell line SMMC-7721, TGF-ß1 induced anti-proliferation by up-regulating the expression of p16 and cellular apoptosis by activating caspase 3 in a Smad4-dependent manner. However, further studies on TGFß1-Smad4 signal system are required to clarify the pathogenesis and molecular mechanism of HCC. Additionally, the knock down of a specific gene using lentiviral-mediated RNAi appears to be a promising tool and strategy for analyzing endogenous gene function.

\section{Acknowledgements}

This study was supported by grants from the Technology Research and Development Foundation of Nanjing Medical University (NY04029, CX2004004), Program of Science and Technology of Nanjing (200801128) and Open Project Program of the State Key Laboratory of Cancer Biology (CBSKL2005009). We thank Dr Jing Zheng (Shanghai GeneChem Co., Ltd, P.R. China) for excellent technical help.

\section{References}

1. Paterson IC, Davies M, Stone A, et al: TGF-beta1 acts as a tumor suppressor of human malignant keratinocytes independently of Smad 4 expression and ligand-induced G(1) arrest. Oncogene 21: 1616-1624, 2002

2. Massague J, Blain SW and Lo RS: TGFbeta signaling in growth control, cancer, and heritable disorders. Cell 103: 295-309, 2000.

3. Shi Y and Massague J: Mechanisms of TGF-beta signaling from cell membrane to the nucleus. Cell 113: 685-700, 2003.

4. Kaminska B, Wesolowska A and Danilkiewicz M: TGF beta signalling and its role in tumour pathogenesis. Acta Biochim Pol 52: 329-337, 2005.

5. Derynck R and Zhang YE: Smad-dependent and Smadindependent pathways in TGF-beta family signalling. Nature 425: 577-584, 2003.
6. Jazag A, Ijichi H, Kanai F, et al: Smad4 silencing in pancreatic cancer cell lines using stable RNA interference and gene expression profiles induced by transforming growth factor-beta. Oncogene 24: 662-671, 2005.

7. Xu L: Regulation of Smad activities. Biochim Biophys Acta 1759: 503-513, 2006.

8. Shirai Y, Kawata S, Tamura S, et al: Plasma transforming growth factor-beta 1 in patients with hepatocellular carcinoma. Comparison with chronic liver diseases. Cancer 73: 2275-2279, 1994.

9. Tsai JF, Jeng JE, Chuang LY, et al: Clinical evaluation of urinary transforming growth factor-betal and serum alpha-fetoprotein as tumour markers of hepatocellular carcinoma. Br J Cancer 75: 1460-1466, 1997

10. Tsai JF, Jeng JE, Chuang LY, Chang WY, Hsieh MY, Lin ZY and Tsai JH: Urinary transforming growth factor-beta 1 in relation to serum alpha-fetoprotein in hepatocellular carcinoma. Scand J Gastroenterol 32: 254-260, 1997.

11. Ji GZ, Wang XH, Miao L, Liu Z, Zhang P, Zhang FM and Yang JB: Role of transforming growth factor-beta1-smad signal transduction pathway in patients with hepatocellular carcinoma. World J Gastroenterol 12: 644-648, 2006.

12. Abou-Shady M, Baer HU, Friess H, et al: Transforming growth factor betas and their signaling receptors in human hepatocellular carcinoma. Am J Surg 177: 209-215, 1999.

13. Idobe Y, Murawaki Y, Kitamura Y and Kawasaki H: Expression of transforming growth factor-beta 1 in hepatocellular carcinoma in comparison with the non-tumor tissue. Hepatogastroenterology 50: 54-59, 2003.

14. Torbenson M, Marinopoulos S, Dang DT, et al: Smad4 overexpression in hepatocellular carcinoma is strongly associated with transforming growth factor beta II receptor immunolabeling. Hum Pathol 33: 871-876, 2002.

15. Kawate S, Takenoshita S, Ohwada S, et al: Mutation analysis of transforming growth factor beta type II receptor, Smad2, and Smad4 in hepatocellular carcinoma. Int J Oncol 14: 127-131, 1999.

16. Ijichi $\mathrm{H}$, Ikenoue $\mathrm{T}$, Kato $\mathrm{N}$, et al: Systematic analysis of the TGF-beta-Smad signaling pathway in gastrointestinal cancer cells. Biochem Biophys Res Commun 289: 350-357, 2001.

17. Li Q, Wu L, Oelschlager DK, et al: Smad4 inhibits tumor growth by inducing apoptosis in estrogen receptor-alpha-positive breast cancer cells. J Biol Chem 280: 27022-27028, 2005.

18. Hahn SA, Schutte M, Hoque AT, et al: DPC4, a candidate tumor suppressor gene at human chromosome 18q21.1. Science 271: 350-353, 1996

19. Losi L, Bouzourene H and Benhattar J: Loss of Smad4 expression predicts liver metastasis in human colorectal cancer. Oncol Rep 17: 1095-1099, 2007.

20. Yakicier MC, Irmak MB, Romano A, Kew M and Ozturk M: Smad2 and Smad4 gene mutations in hepatocellular carcinoma. Oncogene 18: 4879-4883, 1999.

21. Tannapfel A, Anhalt K, Hausermann P, et al: Identification of novel proteins associated with hepatocellular carcinomas using protein microarrays. J Pathol 201: 238-249, 2003.

22. Ji G, Zhang F, Zhai H, Fan Z, Fan D and Wang X: Construction and identification of lentiviral vector of RNA interference of Smad4 gene. J Fourth Mil Med Univ 27: 600-602, 2006.

23. Zhang SW, Lin WS, Ying XL, Zhu D, Guo MY and Gu JX: Effect of suppression of TGF-beta1 expression on cell-cycle and gene expression of beta-1,4-galactosyltransferase 1 in human hepatocarcinoma cells. Biochem Biophys Res Commun 273: 833-838, 2000.

24. Matsuura I, Denissova NG, Wang G, He D, Long J and Liu F: Cyclin-dependent kinases regulate the antiproliferative function of Smads. Nature 430: 226-231, 2004.

25. Hunt KK, Fleming JB, Abramian A, Zhang L, Evans DB and Chiao PJ: Overexpression of the tumor suppressor gene Smad4/DPC4 induces p21waf1 expression and growth inhibition in human carcinoma cells. Cancer Res 58: 5656-5661, 1998.

26. Yanagisawa K, Uchida K, Nagatake M, et al: Heterogeneities in the biological and biochemical functions of Smad2 and Smad4 mutants naturally occurring in human lung cancers. Oncogene 19: 2305-2311, 2000.

27. Lee TK, Man K, Poon RT, Lo CM, Ng IO and Fan ST: Disruption of p53-p21/WAF1 cell cycle pathway contributes to progression and worse clinical outcome of hepatocellular carcinoma. Oncol Rep 12: 25-31, 2004.

28. Hsu LS, Lee HC, Chau GY, Yin PH, Chi CW and Lui WY: Aberrant methylation of $E D N R B$ and $p 16$ genes in hepatocellular carcinoma (HCC) in Taiwan. Oncol Rep 15: 507-511, 2006. 
29. Grau AM, Zhang L, Wang W, et al: Induction of $\mathrm{p} 21$ waf1 expression and growth inhibition by transforming growth factor beta involve the tumor suppressor gene DPC4 in human pancreatic adenocarcinoma cells. Cancer Res 57: 3929-3934, 1997.

30. Ijichi H, Otsuka M, Tateishi K, et al: Smad4-independent regulation of $\mathrm{p} 21 / \mathrm{WAF} 1$ by transforming growth factor-beta. Oncogene 23: 1043-1051, 2004.

31. Wrana JL: Regulation of Smad activity. Cell 100: 189-192, 2000 .
32. Staib F, Hussain SP, Hofseth LJ, Wang XW and Harris CC: TP53 and liver carcinogenesis. Hum Mutat 21: 201-216, 2003.

33. Persad R, Liu C, Wu TT, Houlihan PS, Hamilton SR, Diehl AM and Rashid A: Overexpression of caspase-3 in hepatocellular carcinomas. Mod Pathol 17: 861-867, 2004.

34. Park SY, Kim GY, Bae SJ, Yoo YH and Choi YH: Induction of apoptosis by isothiocyanate sulforaphane in human cervical carcinoma HeLa and hepatocarcinoma HepG2 cells through activation of caspase-3. Oncol Rep 18: 181-187, 2007. 